\title{
Two- and Three-Layered Dissolving Microneedles for Transcutaneous Delivery of Model Vaccine Antigen in Rats
}

\author{
Asuka Ikejiri $^{1}$, Yukako Ito ${ }^{1}$, Seishiro Naito ${ }^{2}$, Kanji Takada $^{1}$ \\ ${ }^{1}$ Department of Pharmacokinetics, Kyoto Pharmaceutical University, Kyoto, Japan; ${ }^{2}$ Department of Safety Research on Blood and \\ Biological Products, National Institute of Infectious Diseases, Tokyo, Japan. \\ Email: asukaikejiri@yahoo.co.jp, takada@mb.kyoto-phu.ac.jp
}

Received March $9^{\text {th }}, 2012$; revised April 15 $5^{\text {th }}, 2012$; accepted May $8^{\text {th }}, 2012$

\begin{abstract}
Purpose: Comparison of transcutaneous immunization of ovalbumin (OA) between two- and three-layered dissolving microneedles (MN) in rats. Methods: We prepared $500 \mu \mathrm{m}$ long two-layered and three-layered dissolving microneedle (2-MN and 3-MN, respectively) arrays from chondroitin sulfate as the base, and OA as the model antigen. The 2-MN containing $\mathrm{OA}$ at the acral portion and 3-MN with $\mathrm{OA}$ at the second portion were administered to rat skin transcutaneously. As a positive control, OA solution was injected subcutaneously (sc). The OA delivery and diffusion in the rat skin were studied using confocal microscopy with fluorescein-conjugated OA (FL-OA). Results: The formulated positions of OA were $0-155 \pm 5 \mu \mathrm{m}$ for 2-MN and $175 \pm 4-225 \pm 5 \mu \mathrm{m}$ for 3-MN. The administered doses of OA were 2.2 $\pm 0.1 \mu \mathrm{g}, 12.0 \pm 0.2 \mu \mathrm{g}$ and $22.0 \pm 0.2 \mu \mathrm{g}$ for $2-\mathrm{MN}, 1.8 \pm 0.2 \mu \mathrm{g}, 12.6 \pm 0.7 \mu \mathrm{g}$, and $20.4 \pm 0.3 \mu \mathrm{g}$ for 3-MN, $10 \mu \mathrm{g}, 100$ $\mu \mathrm{g}$ and $1000 \mu \mathrm{g}$ for sc injection. At 4 weeks after the first administration, 3-MN showed about $2.5-7.0$ fold and 5.4 fold higher total $\operatorname{Ig}(\mathrm{G}+\mathrm{A}+\mathrm{M})$ antibody than 2-MN and sc injection of the OA solution. Conclusions: The 3-MN, which delivered OA to the epidermis, is a useful drug delivery system for transcutaneous antigen delivery.
\end{abstract}

Keywords: Dissolving Microneedles (MNs); Two-Layered MNs; Three-Layered MNs; Transcutaneous Vaccine; Ovalbumin; Fl-Ovalbumin (Fl-OA)

\section{Introduction}

Percutaneous route is an attractive route for the delivery of biopharmaceuticals, because there are many advantages: 1) little or no degradation by hydrolytic enzymes compared to that in the gastrointestinal tract; 2) no firstpass effects of the liver associated with oral delivery; 3 ) less or no pain compared to subcutaneous injection; 4) better convenience of administration than intravenous injection; 5) a better and more continuously controlled delivery rate than those of oral and subcutaneous sustainedrelease preparations; and 6) easy removal if side-effects appear [1,2]. However, transdermal drug delivery systems (TDDSs) are not recognized as a conventional dosage form, because the permeability of drugs through the human skin is limited by the strong barrier function of the skin. Especially, biopharmaceuticals do not permeate through the skin at therapeutically relevant amounts and rates [3]. Vaccine antigens, which are also representative biopharmaceuticals, are used as an injection preparation. To develop an alternative dosage form, not only oral vaccines [4], but also skin-administered vaccines have been sought [5].

Human skin is composed of three layers: the stratum corneum, epidermis, and dermis [1]. The first one is the $10-15 \mu \mathrm{m}$ thick outer layer, which is dead tissue. The second one is the stratum corneum having a strong primary barrier function against exogenous compounds, including drugs. The third barrier is the viable epidermis having the thickness of $100-150 \mu \mathrm{m}$ which contains tissues such as living cells. However, there is no blood vessel in the epidermis. Several approaches such as chemical enhancers, electric fields, ultrasound, and thermal methods have been applied to increase the skin permeability of drugs, [6-10]. In the case of skin vaccine, microparticle systems are studied [11]. However, these TDDSs have not been recognized as a general TDDS, because of the strong barrier function of the skin, which prevents the permeation of drugs through the skin. The application of nanotechnology made it possible to produce microneedles by which the skin permeability of drugs was dramatically increased. Among them, coated microneedle arrays were shown to increase the transdermal permeability of ovalbumin (OA), a model antigen [12]. In addition, Matriano et al. demonstrated that skin immunization was effective by the application of antigen cream after the skin was treated with microneedles, thereby forming microconduits on the skin [13]. 
Those microneedles for percutaneous DDS are classified into three categories: 1) extremely small needles through which a drug solution can be injected into the skin; 2) metallic and/or silastic microneedles onto which a surface drug is coated; and 3) metallic and/or silastic microneedles which form microconduits on the skin, after which a drug solution is applied following removal of the microneedles. These materials - silicon and metals - are not optimal because they are exogenous substances. In contrast to those microneedles, we designed dissolving microneedles with water-soluble thread-forming biopolymers where chondroitin sulfate, dextran, hyaluronic acid, and albumin were used as the base. The drug was formulated as a solid dispersion. As the first step of our research, pen-type dissolving microneedle having $1-2 \mathrm{~mm}$ length and $0.4 \mathrm{~mm}$ diameter were prepared and inserted into the skin. In this case, the base immediately dissolved and the drug was released and absorbed into systemic circulation with high absorption efficiency. By means of dissolving microneedles, high bioavailabilities (BAs) of biopharmaceuticals were obtained, $91.3 \%-97.7 \%$, of insulin in mice [14] and $81.5 \%-102.3 \%$ of low molecular weight heparin (LMWH) in rats [15]. Furthermore, high BAs of $87.5 \%$ were obtained for rhGH in rats [16] and of $82.1 \%$ - 99.4\% for erythropoietin (EPO) in mice [17]. The relative BA of interferon (IFN) against subcutaneous (sc) injection of IFN solution was $79.9 \%-17.8 \%$ in rats [18]. The relative BA of insulin was $90 \%-99 \%$ in dogs [19]. As the second step of our research, we designed a dissolving microneedle array chip, approximately 1.0 $\mathrm{cm}^{2}$, on which $100-225$ dissolving microneedles were formed [20]. Each dissolving microneedle had $500 \mu \mathrm{m}$ length, with $300 \mu \mathrm{m}$ diameter at its base. The drug was formulated at the acral portion of the dissolving microneedle, which was inserted into the skin region of the epidermis and epidermal-dermal junctions by finger pressure. Proof-of-concept experiments using insulin [20], erythropoietin [21], desmopressin [22], and recombinant human growth hormone (rhGH) [22] showed respectively high BAs: $97.9 \% \pm 17.3 \%, 65.9 \%-69.0 \%, 90.0 \%$ - 93.1\%, and $72.8 \%-89.9 \%$. As a subsequent application of dissolving microneedle array chips, OA was used as a model skin vaccine antigen. The skin is densely populated with antigen presenting cells (APCs). Langerhans cells (LCs) and dermal dendritic cells are highly distributed respectively in the epidermis and dermis. Their function is to detect the environment, process antigens and present specific epitopes to T cells. Spontaneously, the dissolving microneedles physically break the barrier function of the skin and deliver the antigen to the skin tissue. However, controversy persists about the antigen target cells. Several groups regard LCs as the target [2325]; other groups see dendritic cells as targets [26,27]. Therefore, two- and three-layered dissolving microneedles were prepared, where OA was formulated at the acral portion of microneedles and at the second portion of microneedles from their tops, respectively. Comparison of the skin vaccination efficiency results is described in this report.

\section{Materials and Methods}

\subsection{Materials}

Ovalbumin (OA) was obtained from Sigma-Aldrich Corp. (St. Louis, MO, USA). Fluorescein labeled OA (FL-OA) was obtained from Molecular Probes Inc. (Eugene, USA). ELISA kits for measuring the OA-specific $\operatorname{Ig}(G+A+M)$ and $\mathrm{IgE}$ antibody concentrations in the rat plasma were obtained from Alpha Diagnostic International Inc. (TX, U.S.A.). An ELISA kit for measuring the OA contents in dissolving microneedles was obtained from Morinaga Institute of Biological Science Inc. (Tokyo, Japan). Sodium chondroitin sulfate was obtained from Nacalai Tesque Inc. (Kyoto, Japan). Brilliant blue (BB) was obtained from Tokyo Chemical Industry Co. Ltd. (Tokyo, Japan). All other materials were of reagent grade and were used as received. Male Brown Norway rats (10 - 12 weeks old) and Male Wistar Hannover rats (11 - 12 weeks old) used in the study were obtained from Japan SLC Inc. (Hamamatsu, Japan). A standard solid-meal commercial food (LabDiet $^{\circledR}$; Nippon Nousan Ltd., Yokohama, Japan) was used. All animal protocols were approved by the institutional animal care and use committee. Experiments were conducted in accordance with the Guidelines for Animal Experimentation, Kyoto Pharmaceutical University.

\subsection{Preparation of Dissolving Microneedle Array Chips and Subcutaneous Solutions Containing OA}

To produce the dense OA solution, hydrogel, 110, 140, or $170 \mu \mathrm{l}$ of distilled water was added to the mixture of 2 $\mathrm{mg}, 10 \mathrm{mg}$, or $20 \mathrm{mg}$ of OA, $0.1 \mathrm{mg}$ of BB and $35 \mathrm{mg}$ of sodium chondroitin sulfate and was kneaded at the low temperature, $15^{\circ} \mathrm{C}$. To produce two-layered microneedle array chips, the hydrogel was degassed under reduced pressure. It was then dispensed into a mold containing 225 inverted cone-shaped wells with an area of $2.25 \mathrm{~cm}^{2}$. Each well had $500 \mu \mathrm{m}$ depth and $300 \mu \mathrm{m}$ diameter at its top. The mold was covered with a $300 \mathrm{~g}$ steel plate; then the drug hydrogel was filled into the wells. After the plate was removed, hydrogel made of $15 \mathrm{mg}$ of chondroitin sulfate and $25 \mathrm{ml}$ of distilled water was painted over the mold. It was then dried under pressure of the stainless steel plate for $3 \mathrm{~h}$. Thereafter, the plate was removed and the dissolving microneedle array chip was obtained by detaching them from a supporting material base. To produce a three-layered microneedle array chip, filling and 
drying were performed in the following order: 1) chondroitin sulfate hydrogel; 2) OA hydrogel; and 3) chondroitin sulfate hydrogel. To make FL-OA microneedle array chip, a mixture of OA and FL-OA (3:1) was used.

The OA solution for subcutaneous injection study was prepared by dissolving $10 \mathrm{mg}, 100 \mathrm{mg}$, or $1000 \mathrm{mg}$ of OA with $200 \mathrm{ml}$ of phosphate buffered saline (PBS).

\subsection{Microscopic Observation of Dissolving Microneedles}

Dissolving microneedle array chips containing FL-OA were observed using a digital video microscope (VH5500; Keyence Co., Osaka, Japan) under normal light and under fluorescent light, $495 \mathrm{~nm}$.

\subsection{OA Content in Dissolving Microneedle Array Chip}

The OA was extracted from dissolving microneedle array chip with $10.0 \mathrm{ml}$ of $0.1 \mathrm{mM}$ phosphate buffer, $\mathrm{pH} 7.4$, and the OA contents were determined using an ELISA kit (Morinaga Institute of Biological Science, Inc., Tokyo, Japan).

\subsection{Immunization Studies in Rats}

Male Brown Norway rats, 10 - 12 weeks old at primary immunization, were anesthetized with intraperitoneal injection of sodium pentobarbital, $50 \mathrm{mg} / \mathrm{kg}$. One group consisted of 4 - 5 rats. At 5 min before administration, $0.5 \mathrm{ml}$ of blank blood samples was obtained from the left jugular vein using a heparinized syringe. Hair on the abdominal region was removed with a shaver (ES7111; Panasonic Inc., Osaka, Japan). The dissolving microneedles were inserted into the skin by pressing the chip with two fingers for 3 min with no treatment. The animals were boosted with the dissolving microneedles at 2 weeks after first immunization. Blood samples, $0.5 \mathrm{ml}$, were obtained from the left jugular vein using a heaprinized syringe at 2 weeks and 4 weeks after the first immunization. By centrifugation at $9000 \times \mathrm{g}$ for $10 \mathrm{~min}$ at $4^{\circ} \mathrm{C}$ using a centrifuge (Kubota 1700; Kubota Corp., Tokyo, Japan), $200 \mu$ plasma samples was obtained. The resultant plasma samples were stored at $-80^{\circ} \mathrm{C}$ until analysis.

For the sc injection experiment, OA solution was injected to the abdominal region of rats at doses of 10, 100, and $1000 \mu \mathrm{g} / \mathrm{rat}$. The animals were boosted with OA solution at 2 weeks after first immunization. At 2 and 4 weeks after first immunization, $0.5 \mathrm{ml}$ of blood samples was obtained from the left jugular vein using a heparinnized syringe.

All these plasma samples were immediately frozen in a deep freezer at $-80^{\circ} \mathrm{C}$ until analysis.

\subsection{Assay for Anti-OA Total $\operatorname{Ig}(G+A+M)$ and IgE Antibody}

The OA-specific $\operatorname{Ig}(\mathrm{G}+\mathrm{A}+\mathrm{M})$ and $\operatorname{IgE}$ antibody concentrations in the rat plasma samples were determined using an ELISA kit (Alpha Diagnostic International, Inc., TX, USA). The concentration of anti-OA antibody of the plasma samples was determined against a standard curve of rat OA Ig calibrators. Plate washers were used (Dia-washer II; Dia Iatron Co. Ltd. USA). Absorbance was measured at $450 \mathrm{~nm}$ using a microplate reader (MTP-300; Corona Electric Co. Ltd., Japan). The rate of change of IgE level at 2 weeks after the start of transcutaneous immunization against the pretreatment level was calculated using the following equation.

\section{Changing rate of IgE level (\%) \\ $=100 \times(\operatorname{IgE}$ level after immunization - IgE level after immunization $) / \operatorname{IgE}$ level before immunization}

\subsection{In Vivo Delivery and Distribution Experiment of FL-OA in the Rat Skin}

Male Wistar Hannover rats, 265 - 305 g, were used. Under anesthesia with an intraperitoneal injection of sodium pentobarbital, $50 \mathrm{mg} / \mathrm{kg}$, the hair covering the abdominal skin was removed using an electric shaver. Dissolving microneedles containing FL-OA was administered to the skin. To study the delivery and diffusion rate of FL-OA to the vertical direction of the rat skin, skin samples were obtained at $30 \mathrm{~s}$ and at 2, 5, and $10 \mathrm{~min}$ after administration of the FL-OA dissolving microneedles to the rat skin. The skin samples were embedded (Histo Prep ${ }^{\mathbb{R}}$; Fisher Scientific International, NJ, USA), frozen at $-80^{\circ} \mathrm{C}$ in a deep-freezer, and sectioned verticality using a microtome (THK; Kenis Ltd., Osaka, Japan). The skin sections, ap- proximately $40 \mu \mathrm{m}$, were mounted on glass slides. The slides of FL-OA loaded rat skin were visualized - under normal light and the fluorescent light, $495 \mathrm{~nm}$, with no staining or treatment - through a $10 \times$ objective using a video microscope (VH-5500; Keyence Co., Osaka, Japan) equipped with a filter for fluorescein observation.

To study the horizontal distribution of FL-OA in the rat skin, skin samples were obtained at 1 min after administration of the two- and three-layered dissolving microneedles containing FL-OA. The skin samples were embedded, frozen at $-80^{\circ} \mathrm{C}$ in a deep-freezer, and sectioned horizontally from the surface to the depth of 400 $\mu \mathrm{m}$ using a cryostat microtome (Leica CM $3050 \mathrm{~S}$; Leica Inc., Nussloch, Germany). From each slice sample, 20 $\mu \mathrm{m}$ thick FL-OA was extracted using $700 \mu \mathrm{l}$ of $0.1 \mathrm{~N}$ 
$\mathrm{NaOH}$ solution. After vortexing for $1 \mathrm{~min}$ and centrifugation at $9000 \times \mathrm{g}$ for $5 \mathrm{~min}, 100 \mu \mathrm{l}$ of supernate was used for measuring fluorescent intensity by Fluoroskan (Ascent CF; Labsystems, Helsinki, Finland), where excitation and emission wavelengths were, respectively, 485 and $538 \mathrm{~nm}$. The distribution ratio of FL-OA in rat skin was calculated using the following equation.

$$
\begin{aligned}
& \text { Disttribution ratio of FL-OA } \\
& =\frac{100 \times \text { fluorescent intensity of each section }}{\text { max imum fluorescent intensity in section }}
\end{aligned}
$$

\subsection{Statistics}

All data are shown as mean \pm S.E. Statistical differences were inferred as significant when $p<0.05$ or $p<0.01$ (Student's unpaired $t$-test).

\section{Results}

\subsection{Physicochemical Properties of Dissolving Microneedles}

The prepared two- and three-layered dissolving microneedles containing FL-OA are presented in Figure 1. In addition, the images of the enlarged dissolving microneedles are presented in Figure 2. As shown in those photos, FL-OA was formulated at the acral or second portions of dissolving microneedles. The physicochemical properties of the prepared dissolving microneedles are shown in Table 1. Mean lengths of the dissolving microneedles were, respectively, $480 \pm 9 \mu \mathrm{m}$ and $485 \pm 7$ $\mu \mathrm{m}$ for two- and three-layered dissolving microneedles. The basement diameters were, respectively, $275 \pm 8 \mu \mathrm{m}$ and $281 \pm 3 \mu \mathrm{m}$. The mean length of the FL-OA loaded space was $0-155 \pm 5 \mu \mathrm{m}$ for two-layered and $175 \pm 4$ $224 \pm 5 \mu \mathrm{m}$ for three-layered dissolving microneedles.

\subsection{In Vivo Immunological Evaluation of Dissolving Microneedles in Rats}

The obtained dissolving microneedles containing OA were administered to the rat skin by pressing the chips on the abdominal skin where the hair had been removed. After administration, the chips were recovered and the amounts of $\mathrm{OA}$ in the recovered chips were measured. Table 2 presents the OA contents in the chips and recovered amounts of OA in the chips.

In the case of two-layered OA microneedle array chips, the OA contents were, respectively, $2.2 \pm 0.4,12.6 \pm 2.4$, and $22.7 \pm 1.3 \mu \mathrm{g}$. In contrast, the recovered $\mathrm{OA}$ in the chips were, respectively, $0.1 \pm 0.1,0.5 \pm 0.2$, and $0.7 \pm$ $0.2 \mu \mathrm{g}$. By subtracting the recovered amount of OA from the amount of OA formulated in microneedle array chips, the administered doses of OA were estimated as $2.2 \pm 0.1$, $12.0 \pm 0.2$, and $22.0 \pm 0.2 \mu \mathrm{g}$, respectively, as also shown
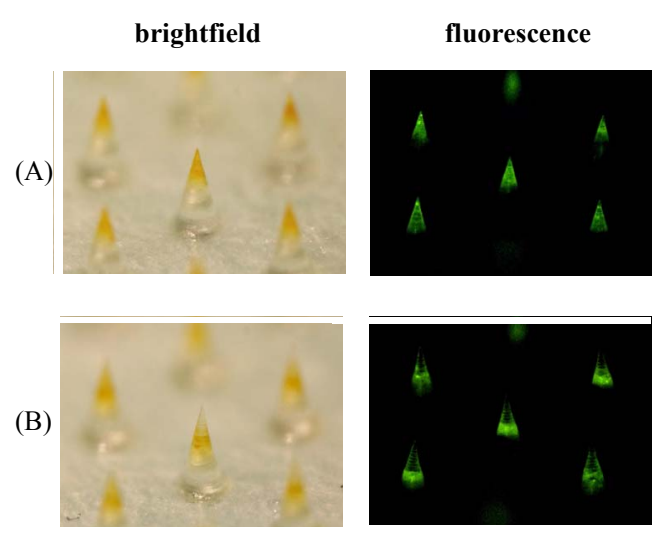

Figure 1. Fluorescence microscopical images of the (A) twoand (B) three-layered FL-OA dissolving microneedles made of chondroitin sulfate as the base used in the in vivo delivery and distribution experiments. Left panels portray overview images of dissolving microneedles under normal light.

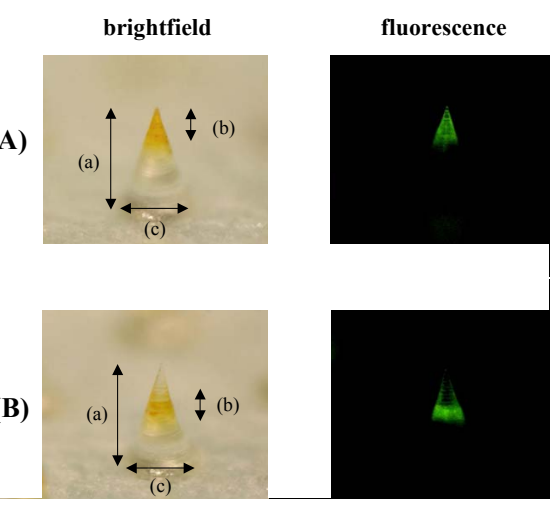

Figure 2. Fluorescence microscopy of enlarged image of the (A) two- and (B) three-layered FL-OA dissolving microneedles. Left panels portray overview images of dissolving microneedles under normal light. Arrows indicate the following lengths: (a) Whole length; (b) Length of the FL-OA space; (c) Diameter of the basement.

Table 1. Physicochemical property of two- and three-layered dissolving microneedles.

\begin{tabular}{cccc}
\hline $\begin{array}{c}\text { Microneedle } \\
\text { array }\end{array}$ & $\begin{array}{c}\text { Height (a) } \\
(\boldsymbol{\mu} \mathbf{m})\end{array}$ & $\begin{array}{c}\text { Length of FL-OA } \\
\text { space }(\mathbf{b})^{*}(\boldsymbol{\mu m})\end{array}$ & $\begin{array}{c}\text { Diameter at } \\
\text { basement }(\mathbf{c})^{*}(\boldsymbol{m})\end{array}$ \\
\hline $\begin{array}{c}\text { Two-layered } \\
\text { microneedles }\end{array}$ & $480 \pm 9$ & $0-155 \pm 5$ & $275 \pm 8$ \\
$\begin{array}{l}\text { Three-layered } \\
\text { microneedles }\end{array}$ & $485 \pm 7$ & $175 \pm 4-255 \pm 5$ & $281 \pm 3$ \\
\hline
\end{tabular}

Each values show the mean \pm S.E. $(n=6) .{ }^{*}$ The alphabets inside the parentheses represent the corresponding length in Figure 2.

\section{in Table 2 .}

In the case of three-layered OA microneedle array chips, the OA contents were, respectively, $2.2 \pm 0.3,13.7$ \pm 0.5 , and $21.1 \pm 1.6 \mu \mathrm{g}$. However, the recovered $\mathrm{OA}$ contents in the chips were, respectively, $0.5 \pm 0.2,1.0 \pm$ 0.8 , and $0.7 \pm 0.3 \mu \mathrm{g}$. By subtracting the recovered amount of OA from the amount of OA formulated in 
Table 2. OA contents in dissolving microneedle array chip, recovered amount of $\mathrm{OA}$ and administered dose.

\begin{tabular}{|c|c|c|c|}
\hline Formulation & $\begin{array}{l}\text { OA content } \\
(\mu \mathrm{g} / \mathrm{MNs})\end{array}$ & $\begin{array}{c}\text { Recovered amount } \\
\text { of OA }(\mu \mathrm{g} / \mathrm{MNs})\end{array}$ & $\begin{array}{c}\text { Administered } \\
\text { dose }(\mu \mathrm{g} / \mathrm{rat})\end{array}$ \\
\hline \multirow{3}{*}{$\begin{array}{l}\text { Two-layered } \\
\text { microneedles }\end{array}$} & $2.2 \pm 0.4$ & $0.1 \pm 0.1$ & $2.2 \pm 0.1$ \\
\hline & $12.6 \pm 2.4$ & $0.5 \pm 0.2$ & $12.0 \pm 0.2$ \\
\hline & $22.7 \pm 1.3$ & $0.7 \pm 0.2$ & $22.0 \pm 0.2$ \\
\hline \multirow{3}{*}{$\begin{array}{l}\text { Three-layered } \\
\text { microneedles }\end{array}$} & $2.2 \pm 0.3$ & $0.5 \pm 0.2$ & $1.8 \pm 0.2$ \\
\hline & $13.7 \pm 0.5$ & $1.0 \pm 0.8$ & $12.6 \pm 0.7$ \\
\hline & $21.1 \pm 1.6$ & $0.7 \pm 0.3$ & $20.4 \pm 0.3$ \\
\hline \multirow{3}{*}{$\begin{array}{l}\text { s.c. injection } \\
\text { solution }\end{array}$} & - & - & $10^{*}$ \\
\hline & - & - & $100^{*}$ \\
\hline & - & - & $1000^{*}$ \\
\hline $\begin{array}{c}\text { Placebo } \\
\text { microneedles }\end{array}$ & - & - & - \\
\hline
\end{tabular}

Each values show the mean \pm S.E. $(n=4-10),{ }^{*}$ injected dose.

microneedle array chips, the administered doses of OA were estimated respectively as $1.8 \pm 0.2,12.6 \pm 0.7$, and $20.4 \pm 0.3 \mu \mathrm{g}$.

In Table 3 and Figure 3, the anti- $\operatorname{Ig}(G+A+M)$ concentrations are shown after application of two- and three-layered dissolving microneedles and sc injection of $\mathrm{OA}$ solution. When OA solutions were injected to the rats at three different doses of 10,100 , and $1000 \mu \mathrm{g}$, the anti- $\operatorname{Ig}(G+A+M)$ concentrations at 2 weeks after the first immunization were, respectively, $2.0 \pm 0.8,4.7 \pm 1.1$, and $9.7 \pm 3.1\left(\times 10^{4} \mathrm{U} / \mathrm{mL}\right)$. At 2 weeks after the second immunization, the anti- $\operatorname{Ig}(\mathrm{G}+\mathrm{A}+\mathrm{M})$ concentrations were more increased, to $67.1 \pm 23.5,124.0 \pm 38.1$, and $164.0 \pm 30.0\left(\times 10^{4} \mathrm{U} / \mathrm{mL}\right)$, respectively.

After application of the two-layered dissolving microneedles, the anti- $\operatorname{Ig}(\mathrm{G}+\mathrm{A}+\mathrm{M})$ concentrations at 2 weeks after the first immunization were, respectively, 3.2 $\pm 0.3,2.5 \pm 0.5$ and $2.8 \pm 0.6\left(\times 10^{4} \mathrm{U} / \mathrm{mL}\right)$. At 2 weeks after the second immunization, the anti- $\operatorname{Ig}(\mathrm{G}+\mathrm{A}+\mathrm{M})$ concentrations were, respectively, $39.8 \pm 13.3,145.6 \pm$ 25.8 , and $70.3 \pm 39.2\left(\times 10^{4} \mathrm{U} / \mathrm{mL}\right)$.

Three-layered dissolving microneedles showed higher plasma $\operatorname{Ig}(\mathrm{G}+\mathrm{A}+\mathrm{M})$ levels at both 2 and 4 weeks. The anti- $\operatorname{Ig}(G+A+M)$ concentrations at 2 weeks after the first immunization were, respectively, $16.5 \pm 1.9$, $13.0 \pm 4.3$, and $14.4 \pm 0.6\left(\times 10^{4} \mathrm{U} / \mathrm{mL}\right)$. At 2 weeks after the second immunization, the anti- $\operatorname{Ig}(\mathrm{G}+\mathrm{A}+\mathrm{M})$ concentrations were, respectively, $282.8 \pm 78.5,368.4 \pm 42.5$, and $394.6 \pm 45.6\left(\times 10^{4} \mathrm{U} / \mathrm{mL}\right)$. At 4 weeks after the first administration, three-layered microneedles showed about $2.5-7.0$ fold and 5.4 fold higher total $\operatorname{Ig}(G+A+M)$ antibody levels than either the two-layered microneedles or sc injection solution.

Using plasma samples obtained at 2 weeks after the start of the experiment and the pre-dose plasma samples,
Table 3. Administered dose of $\mathrm{OA}$ and total $\operatorname{Ig}(\mathrm{G}+\mathrm{A}+\mathrm{M})$ antibody.

\begin{tabular}{cccc}
\hline Formulation & $\begin{array}{c}\text { Administered } \\
\text { dose }(\mu \mathrm{g} / \mathbf{r a t})\end{array}$ & $\begin{array}{c}\text { Total Ig(GAM) } \\
\text { after 2 weeks } \\
\times \mathbf{1 0}^{4}(\mathbf{U} / \mathbf{m L})\end{array}$ & $\begin{array}{c}\text { Total Ig(GAM) } \\
\text { after 4 weeks } \\
\times \mathbf{1 0}^{4}(\mathbf{U} / \mathbf{m L})\end{array}$ \\
\hline $\begin{array}{c}\text { Two-layered } \\
\text { microneedles }\end{array}$ & $2.2 \pm 0.1$ & $3.2 \pm 0.3$ & $39.8 \pm 13.3$ \\
& $12.0 \pm 0.2$ & $2.5 \pm 0.5$ & $145.6 \pm 25.8$ \\
& $22.0 \pm 0.2$ & $2.8 \pm 0.6$ & $70.3 \pm 39.2$ \\
Three-layered & $1.8 \pm 0.2$ & $16.5 \pm 1.9^{\mathrm{a}}$ & $282.8 \pm 78.5^{\mathrm{b}}$ \\
microneedles & $12.6 \pm 0.7$ & $13.0 \pm 4.3$ & $368.4 \pm 42.5^{\mathrm{a}}$ \\
& $20.4 \pm 0.3$ & $14.4 \pm 0.6^{\mathrm{a}}$ & $394.6 \pm 45.6^{\mathrm{a}}$ \\
s.c. injection & $10^{*}$ & $2.0 \pm 0.8$ & $67.1 \pm 23.5$ \\
solution & $100^{*}$ & $4.7 \pm 1.1$ & $124.0 \pm 38.1$ \\
$\begin{array}{c}\text { Placebo } \\
\text { microneedles }\end{array}$ & $1000^{*}$ & $9.7 \pm 3.1$ & $164.0 \pm 30.0$ \\
\hline
\end{tabular}

Each values show the mean \pm S.E. $(n=4-10) ;{ }^{*}$ Injected dose; ${ }^{\mathrm{a}, \mathrm{b}}$ Compared to two-layered OA microneedles group at the corresponding dose $\left({ }^{\mathrm{a}} p<0.01\right.$, $\left.{ }^{\mathrm{b}} p<0.05\right)$.

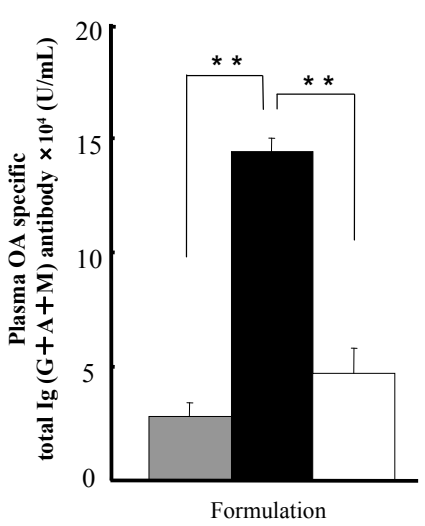

(a)

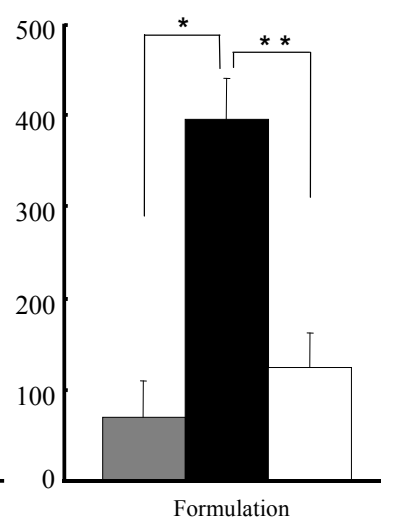

(b)
Figure 3. Plasma OA specific total Ig $(G+A+M)$ antibody concentrations at (a) 2 and (b) 4 weeks post first immunization after transcutaneous administration of two- and threelayered dissolving microneedles containing $\mathrm{OA}$ to the abdominal rat skin. Plasma OA specific total $\operatorname{Ig}(\mathbf{G}+\mathbf{A}+\mathbf{M})$ antibody concentrations were measured using ELISA. The gray bars denote antibody concentrations obtained after administration of two-layered $\mathrm{OA}$ dissolving microneedles, $22.0 \pm 0.2 \mu \mathrm{g} / \mathrm{rat}$. The black bars denote those obtained after administration of three-layered $\mathrm{OA}$ dissolving microneedles, $20.4 \pm 0.3 \mu \mathrm{g} / \mathrm{rat}$. The white bars denote those obtained after subcutaneous administration of OA: $100 \mu \mathrm{g} / \mathrm{rat}$. Each value shows the mean \pm S.E. of 4 - 5 experiments. ${ }^{*} p<0.05,{ }^{* * *} p<$ 0.01: significantly different from three-layered microneedles. Each value shows the mean \pm S.E. $(n=4$ - 5).

anti-OA IgE levels were measured. The results are shown in Table 4. The IgE concentrations, after the administration of placebo microneedles, made without OA, and two-layered microneedles $(12.0 \pm 0.2,22.0 \pm 0.2 \mu \mathrm{g} / \mathrm{rat})$, three-layered microneedles $(12.6 \pm 0.7,20.4 \pm 0.3 \mu \mathrm{g} / \mathrm{rat})$ 
Table 4. Plasma anti-OA IgE levels and the changing rate of IgE level at 2 weeks after the start of transcutaneous immunization against the pretreatment level.

\begin{tabular}{ccccc}
\hline \multirow{2}{*}{ Formulation } & \multirow{2}{*}{ Administered dose $(\mu \mathbf{g} / \mathbf{r a t})$} & \multicolumn{2}{c}{ IgE level $(\mathrm{U} / \mathbf{m L})$} & \multirow{2}{*}{ Changing rate of IgE level (\%) } \\
\cline { 3 - 4 } & & $\mathbf{0 ~ w e e k}$ & $\mathbf{2}$ week & $-1.7 \pm 2.3$ \\
\hline Placebo microneedles & - & $51.9 \pm 0.9$ & $50.9 \pm 0.5$ & $-4.7 \pm 2.0$ \\
Two-layered microneedles & $12.0 \pm 0.2$ & $58.9 \pm 0.2$ & $56.1 \pm 1.2$ & $5.8 \pm 1.9$ \\
& $22.0 \pm 0.2$ & $57.4 \pm 1.9$ & $59.9 \pm 0.9$ & $-5.4 \pm 4.0$ \\
Three-layered microneedles & $12.6 \pm 0.7$ & $61.7 \pm 0.6$ & $58.2 \pm 1.9$ & $-6.3 \pm 2.5$ \\
s.c. injection solution & $20.4 \pm 0.3$ & $60.2 \pm 1.1$ & $56.4 \pm 0.8$ & $17.0 \pm 1.6^{\mathrm{a}}$ \\
\hline
\end{tabular}

Changing rate; $100 \times(\operatorname{IgE}$ level after immunization $-\operatorname{IgE}$ level before immunization $) / \operatorname{IgE}$ level before immunization injected dose, ${ }^{\mathrm{a}} p<0.01$, significantly different placebo microneedles preparation; Each values show the mean \pm S.E. $(n=4-10)$.

and sc, were, respectively, $50.9 \pm 0.5,56.1 \pm 1.3,59.9 \pm$ $0.9,58.2 \pm 1.9,56.37 \pm 0.8$, and $63.1 \pm 1.0(\mathrm{U} / \mathrm{mL})$. In contrast, the $\operatorname{IgE}$ concentrations before immunization were, respectively, $51.9 \pm 0.9,58.9 \pm 0.2,57.4 \pm 1.9$, $61.7 \pm 0.6,60.2 \pm 1.1$, and $53.9 \pm 1.0(\mathrm{U} / \mathrm{mL})$. In addition, the estimated rates of change of $\mathrm{IgE}$ level were, respectively, $-1.7 \% \pm 2.3 \%,-4.7 \% \pm 2.0 \%, 5.8 \% \pm 1.9 \%$, $-5.4 \% \pm 4.0 \%,-6.3 \% \pm 2.5 \%$, and $17.0 \% \pm 1.6 \%$. No significant difference was found in the rate of change of anti-OA IgE level between placebo, two-layered and threelayered groups, although the sc injection group showed a significantly higher level than the placebo group.

\subsection{Delivery and Distribution Study in Rat Skin}

The delivery site and the diffusion characteristics of the vaccine antigen in the rat skin were studied by administering the two- or three-layered microneedles containing FL-OA used as a model antigen.

Figure 4 portrays normal and fluorescent images of rat skin sections obtained after the administration of FL-OA loaded dissolving microneedles to the rat skin. In the case of two-layered microneedles containing FL-OA, the spots were detected around the first $200 \mu \mathrm{m}$ of rat skin. However, the spots of green fluorescein, which had been delivered from three-layered microneedles containing FL-OA, were detected mainly from the surface to the first $100 \mu \mathrm{m}$ of the skin. As the figures show, the green fluorescence derived from FL-OA was apparent immediately after its administration; it diffused as time passed.

Because chondroitin sulfate, a water-soluble threadforming polymer, was used as the base polymer to prepare dissolving microneedles, the base dissolved rapidly. Consequently, the release of green fluorescein occurred immediately after administration. Even at $30 \mathrm{~s}$ after administration, the conical shape of dissolving microneedles was not detected completely, although spots of green fluorescein were detected. At 2 and 5 min after administration, green fluorescence spots enlarged transversally. Then diffusion to the transverse direction reached the steady state at $10 \mathrm{~min}$.

Figure 5 presents the horizontal distribution profiles of fluorescent intensity attributable to FL-OA delivered in the rat skin after administration by dissolving microneedles. In the case of two-layered microneedles containing FL-OA, the maximum fluorescent intensity was detected at the first $120 \mu \mathrm{m}$ of rat skin. The distribution profile showed a gradual increase in fluorescence intensity with an increase in the depth from surface to $120 \mu \mathrm{m}$, and a gradual decrease in it with an increase deep from $120 \mu \mathrm{m}$ onward. Furthermore, at the depth of $160-260$ $\mu \mathrm{m}$, the fluorescence intensity distribution was considerably higher than that obtained from three-layered microneedles. The maximum fluorescence intensity obtained after the administration of three-layered microneedles was detected at the first $20 \mu \mathrm{m}$ of the skin. The fluorescence intensity decreased gradually with increased depth. At this depth, distribution of fluorescence intensity was markedly higher than that obtained using two-layered microneedles. The figures show that the two- and three-layered microneedles respectively delivered the model vaccine antigen mainly to the dermal and epidermal layers.

\section{Discussion}

The main function of skin is to provide a protective cover against the hostile external environment: a role that includes immunoprotection. Human skin comprises three layers: the stratum corneum, epidermis, and dermis. The first one is the $10-15 \mu \mathrm{m}$ thick outer layer, which is dead tissue. The stratum corneum is a strong primary barrier against exogenous compounds, including drugs. The second barrier is the viable epidermis, $100-150 \mu \mathrm{m}$, which contains tissues such as living cells. However, the epidermis has no blood vessels. The epidermis primarily comprises keratinocytes, but it also has, distributed amongst the viable keratinocytes, a large population of cells, i.e. $1 \%$ - 3\% of epidermal cells [28], that are involved in immune surveillance, the LCs. Although LCs 


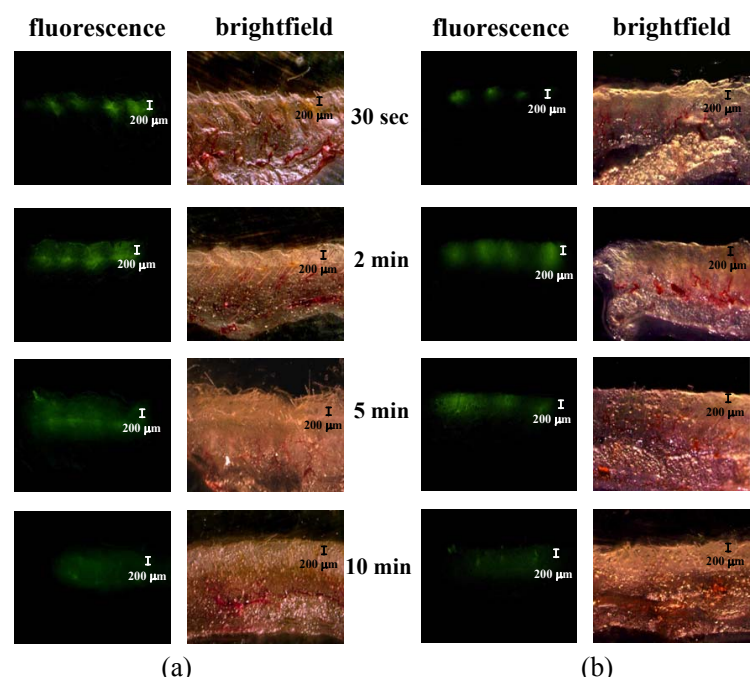

Figure 4. Fluorescence microscopy of the skin of rats who received the (a) two- and (b) three-layered dissolving microneedles containing FL-OA, as visualized through a $50 \times$ objective (scale bar: $200 \mu \mathrm{m}$ ). Dissolving microneedles of two types were administered to the rat abdominal skin. Thereafter, skin tissue samples were obtained at $30 \mathrm{~s}$ and 2, 5 , and $10 \mathrm{~min}$. Right panels show the corresponding brightfield microscopy images.

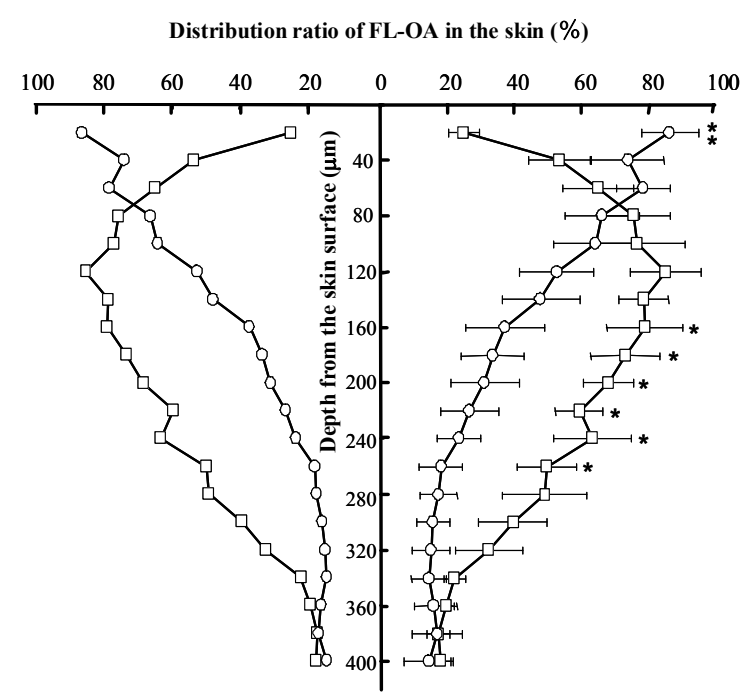

Figure 5. Distribution profiles of FL-OA in the rat skin after administration by dissolving microneedles. Dissolving microneedles of two types were administered to the rat skin. Skin tissue samples were obtained at $1 \mathrm{~min}$. The $20 \mu \mathrm{m}$ thick slice samples were obtained and FL-OA contents in each slice were determined spectrofluorometrically. The distribution ratio of FL-OA in rat skin was calculated using $100 \times$ fluorescent intensity of each section/maximum fluorescent intensity in all sections. The open squares show data obtained after administration of two-layered dissolving microneedles containing FL-OA. The open circles represent data for three-layered dissolving microneedles. ${ }^{* * *} p<0.01$; significantly different from two-layered microneedles. " $p<$ 0.05 ; significantly different from three-layered microneedles. Each value shows the mean \pm S.E. $(n=5)$. are few among the cells in the skin, they account for $25 \%$ of the total skin surface area in humans [29]. In fact, LCs represent an extensive, superficial network barrier of immune cells that make an attractive target for vaccine delivery. The LCs are bone marrow-derived dendritic cells that migrate to epithelial surfaces such as the skin where they perform immunosurveillance [30]. Under normal circumstances, the baseline traffic of LCs exists from the skin to the draining lymph nodes, where the cells present the antigens they have encountered in the epidermis. To increase the skin permeability of drugs, numerous approaches have been attempted using chemical enhancers, electric fields, ultrasound, and thermal methods [6-10]. However, these TDDS' success has been limited because of the strong barrier function of the skin: the low membrane permeability of drugs through the skin. In our earlier studies, two-layered dissolving microneedles with water-soluble thread-forming biopolymers such as chondroitin sulfate, dextran, hyaluronic acid and albumin used as the base were evaluated as a new TDDS. The drug was formulated as a solid dispersion. After administration to the rat skin, high BAs of $91.3 \%-97.7 \%$ were obtained for insulin in mice [14] and of $81.5 \%-102.3 \%$ for LMWH in rats [15]. Furthermore, high BAs of $87.5 \%$ were obtained for rhGH in rats [16] and of $82.1 \%$ $99.4 \%$ for EPO in mice [17]. The relative BA of IFN against sc injection of IFN solution was 79.9\% - 117.8\% in rats [18]. The relative BA of insulin was $90 \%-99 \%$ in dogs [19]. Based on those outcomes, dissolving microneedles have been applied for transcutaneous administration of vaccine antigen. In previous studies of transcutaneous immunization, the mouse model system has been used because many immunological assay systems have been developed and are now commercially available. However, the mouse model is not useful for the study of transcutaneoous immunization, a so-called skin vaccine, because the histology of mouse and human skin differs considerably. According to a report by Monteiro-Riviere et al., the epidermal thickness at the skin of the ventral abdomen is $11.58 \pm 1.02 \mu \mathrm{m}$ for rats and $22.47 \pm 2.40$ $\mu \mathrm{m}$ for dogs [31]. For human skin, the epidermal thickness is reportedly to be $60.3 \pm 15.0 \mu \mathrm{m}$ [32]. It is very difficult to deliver vaccine antigen strictly relying upon the epidermis and/or dermis for any delivery system in mice. Therefore, in this study, we introduced a rat model system. In this study, we used Brown Norway rats because Brown Norway rats have higher sensitivity to OA than Hooded Lister, Piebald Virol Glaxo, and Wistar rats have [33]. In the case of abdominal skin in rats, the respective thicknesses of the stratum corneum, epidermis, and dermis have been reported as $4.56 \pm 0.61 \mu \mathrm{m}, 11.58$ $\pm 1.02 \mu \mathrm{m}$, and $402.0 \pm 49.86 \mu \mathrm{m}[31,34]$. Although a difference exists in the thickness and histology of the skin between rats and humans, it is possible to deliver 
vaccine antigen to the epidermis and/or dermis using dissolving microneedle array chips. Gelinck et al. used an intradermal injection syringe (BD Micro-fine $0.5 \mathrm{~mL}$ U-100 insulin syringe; Microfine Materials Technologies Pte. Ltd.) to administer influenza vaccine antigen to human subjects [35]. However, it is difficult to control the depth of the injection site of vaccine antigen solution inside the skin. Therefore, to perform this study, we designed dissolving microneedles of two types: two-layered and three-layered dissolving microneedles. In the case of two-layered microneedles, OA, the model antigen, was formulated in the acral portion of microneedles, but OA was formulated into the second layer of three-layered microneedles. For two-layered microneedles, the length of the OA formulated layer was $155 \pm 5 \mu \mathrm{m}$ from the top of the microneedles. In the case of three-layered microneedles, OA was formulated at the position of $175 \pm 4 \mu \mathrm{m}$ to $225 \pm 5 \mu \mathrm{m}$ from the top of the microneedles. The epidermal thickness was reported as $11.58 \pm 1.02 \mu \mathrm{m}$ in rats and the layer of dermis is $402.0 \pm 49.86 \mu \mathrm{m}[31,34]$. Consequently, OA would be delivered to the subcutaneous tissue of the rat if the microneedle of $500 \mu \mathrm{m}$ length were inserted completely into the rat skin. However, the full length of the microneedles was not inserted into the skin, as our studies of dissolving microneedles conducted for over a decade have confirmed. Our previous report showed that about a half-length of the microneedles was inserted into the skin [36]. Furthermore, when we inserted $500 \mu \mathrm{m}$ length dissolving microneedles into the skin, no bleeding occurred. Those results suggested that the acral portion of dissolving microneedles was dissolved during passage through the skin. The width of the top portion of dissolving microneedles was approximately $10 \mu \mathrm{m}$. When the acral portion of dissolving microneedles was inserted into the skin, the dissolving microneedles encountered environmental water and dissolved during passage through the epidermis, because Caspers et al. reported that the water content in the skin epidermal layer was as high as $70 \%$ [37]. Fluorescent microscopical experiments using two-layered and threelayered dissolving microneedles containing FL-OA in this study showed that two-layered microneedles delivered FL-OA mainly to the rat skin dermal layer, where dermal DCs are highly distributed, and three-layered microneedles delivered FL-OA mainly to the epidermal layer where LCs are highly distributed. Some controversy persists about the target cells of the transcutaneously delivered antigen. Several groups support LCs. Other groups support dermal DCs. However, this argument remains unresolved, because no device can deliver vaccine antigen to a specific site of the skin, dermis and/or epidermis. Therefore, two- and three-layered dissolving microneedles were prepared. These devices delivered vaccine antigen strictly to the dermis and epider- mis. Before our study of transcutaneous immunization, coated microneedle array chips were applied to study the effect of delivery parameters on immunization to OA that was coated onto the surface of microneedles [38]. For the coated microneedles, the distribution of OA in the rat skin and the antibody responses were independent of the microneedle length used for the delivery of OA, antigen. Moreover, it was shown to be very difficult to control the coating length from the top of the microneedles. Furthermore, in the case of coating microneedles, a vaccine antigen was coated onto the surface of the acral portion of microneedles. In this case, the amount of antigen was limited. In other words, the coating amount of vaccine antigen on the acral surface of microneedles, which was made of steel or a biopolymer-like polylactic acid, is limited. When steel microneedles are used as the device to administer vaccine transcutaneously, a problem exists in terms of its safety: allergy to the steel. To study the safety of OA-loaded dissolving microneedles, plasma $\mathrm{IgE}$ levels were measured at 2 weeks after the start of immunization. In addition, a histological study of the skin tissue was performed after the administration of microneedle array chips. Results showed no significant difference in the rate of increase of IgE levels between active groups and control groups who received placebo microneedles. In our previous studies of the evaluation of the dissolving microneedles containing insulin, EPO and $\mathrm{rhGH}$, no irritation or damage was found at the administered skin tissue at $2 \mathrm{hr}$ and $24 \mathrm{hr}$ after administration [20, 22]. In this study, the rat skin was monitored for 1 month. However, no irritation or damage was found in the skin tissue over that long term of 1 month. Therefore, we infer that dissolving microneedles are a safe transcutaneous drug delivery system for use in immunization.

\section{Conclusion}

This feasibility study of OA loaded two- and three-layered dissolving microneedles having $500 \mu \mathrm{m}$ length and $300 \mu \mathrm{m}$ diameters of their basements was performed using rats. The dissolving microneedles were prepared with chondroitin sulfate as the base polymer using microfabrication technology. The delivery of model antigen, OA, to the epidermis and dermis was ascertained using threelayered and two-layered dissolving microneedles, where OA was formulated respectively as a solid dispersion with chondroitin sulfate at the second portion, $175 \pm 4$ $225 \pm 5 \mu \mathrm{m}$, and acral portion, $0-155 \pm 5 \mu \mathrm{m}$, of microneedles from their tops. Systemic immune responses, OA-specific $\operatorname{Ig}(\mathrm{G}+\mathrm{A}+\mathrm{M})$ antibody in plasma, were 2.5 - 7 fold increased by three-layered dissolving microneedles compared to two-layered dissolving microneedles. Histological studies using FL-OA loaded dissolving microneedles revealed that FL-OA was delivered mainly to the epidermal region of the rat skin, around the 
first $100 \mu \mathrm{m}$ of the skin, by the three-layered dissolving microneedles. Those results revealed that the delivery site of transcutaneous antigen by three-layered dissolving microneedles was the epidermis of the skin where LCs are highly distributed.

\section{Acknowledgements}

This study was supported by a Grant-in-Aid for scientific Research (b) (223100820001) from Ministry of Education, Science, Sports and Culture of Japan, MEXT. This study was also supported by a strategic fund of MEXT from 2008 to 2013 for establishing research foundation in private universities of Japan.

\section{REFERENCES}

[1] J. C. Birchall, "Stratum Corneum Bypassed or Removed," In: E. Touitou and B. W. Barry, Eds., Enhancement in Drug Delivery, CRC Press, New York, 2007, pp. 337351.

[2] M. A. F. Kendall, "Needle-Free Vaccine Injection," In: M. Schafer-Korting, Ed., Drug DelivSery, Handbook of Experimental Pharmacology, Springer Press, Berlin, 2010, pp. 193-220.

[3] G. Walsh, "Biopharmaceuticals," Wiley, West Sussex, 2003.

[4] J. F. S. Mann, V. A. Ferro, A. B. Mullen, L. Tetley, M. Mullen, K. C. Carter, J. Alexander and W. H. Stimsan, "Optimisation of a Lipid Based Oral Delivery System Containing A/Panama Influenza Hemagglutinin," Vaccine, Vol. 22, No. 19, 2004, pp. 2425-2429. doi:10.1016/j.vaccine.2003.11.067

[5] S. Naito, J. Maeyama, T. Mizukami, M. Takahashi, I. Hamaguchi and K. Yamaguchi, "Transcutaneous Immunization by Merely Prolonging the Duration of Antigen Presence on the Skin of Mice Induces a Potent AntigenSpecific Antibody Response Even in the Absence of an Adjuvant," Vaccine, Vol. 25, No. 52, 2007, pp. 87628770. doi:10.1016/j.vaccine.2007.10.031

[6] B. Barry and A. Williams, "Penetration Enhancers," Advanced Drug Delivery Reviews, Vol. 56, No. 5, 2004, pp. 603-618. doi:10.1016/j.addr.2003.10.025

[7] G. Cevc, "Lipid Vesicles and Other Colloids as Drug Carriers on the Skin," Advanced Drug Delivery Reviews, Vol. 56, No. 5, 2004, pp. 675-711. doi:10.1016/j.addr.2003.10.028

[8] L. V. Preat and R. Vanbever, "Skin Electroporation for Transdermal and Topical Delivery," Advanced Drug Delivery Reviews, Vol. 56, No. 5, 2004, pp. 659-674. doi:10.1016/j.addr.2003.10.027

[9] S. Mitragotri and J. Kost, "Low-Frequency Sonophoresis: A Review," Advanced Drug Delivery Reviews, Vol. 56, No. 5, 2004, pp. 589-601. doi:10.1016/j.addr.2003.10.024

[10] A. Doukas, "Transdermal Delivery with a Pressure Wave," Advanced Drug Delivery Reviews, Vol. 56, No. 5, 2004, pp. 559-579. doi:10.1016/j.addr.2003.10.031
[11] S. A. Coulman, A. Anstey, C. Gately, A. Morrissey, P. McLoughlin, C. Allender and J. C. Birchall, "Microneedle Mediated Delivery of Nanoparticles into Human Skin," International Journal of Pharmaceutical, Vol. 366, No. 1-2, 2009, pp. 190-200. doi:10.1016/j.ijpharm.2008.08.040

[12] Q. Zhu, V. G. Zarnitsyn, L. Ye, Z. Wen, Y. Gao, L. Pan, I. Skountzou, H. S. Gill, M. R. Prausnitz, C. Yang and R. W. Compans, "Immunization by Vaccine-Coated Microneedle Arrays Protects Against Influenza Virus Challenge," Proceedings of the National Academy of Sciences of the United States of America, Vol. 106, No. 19, 2009, pp. 7968-7973. doi:10.1073/pnas.0812652106

[13] J. A. Matriano, M. Cormier, J. Johnson, W. A. Young, M. Buttery, Nyam K and P. E. Daddona, "Macroflux ${ }^{\mathbb{B}} \mathrm{Mi}-$ croprojection Array Patch Technology: A New and Efficient Approach for Intracutaneous Immunization," Pharmaceutical Research, Vol. 19, No. 1, 2002, pp. 63-70. doi:10.1023/A:1013607400040

[14] Y. Ito, E. Hagiwara, A. Saeki, N. Sugioka and K. Takada, "Feasibility of Microneedles for Percutaneous Absorption of Insulin," European Journal of Pharmaceutical Sciences, Vol. 29, No. 1, 2006, pp. 82-88. doi:10.1016/j.ejps.2006.05.011

[15] Y. Ito, A. Murakami, T. Maeda, N. Sugioka and K. Takada, "Evaluation of Self-Dissolving Needles Containing Low Molecular Weight Heparin (LMWH) in Rats," Intarnational Journal and Pharmaceutics, Vol. 349, No. 1-2, 2008, pp. 124-129. doi:10.1016/j.ijpharm.2007.07.036

[16] Y. Ito, Y. Ohashi, K. K. Shiroyama, N. Sugioka and K. Takada, "Self-Dissolving Micropiles for the Percutaneous Absorption of Human Growth Hormone in Rats," Biological and Pharmaceutical Bulletin, Vol. 31, No. 8, 2008, pp.1631-1633. doi:10.1248/bpb.31.1631

[17] Y. Ito, J. Yoshimitsu, K. Shiroyama, N. Sugioka and K. Takada, "Self-Dissolving Microneedles for the Percutaneous Absorption of EPO in Mice," Journal of Drug Targeteting, Vol. 14, 2006, pp. 255-262. doi:10.1080/10611860600785080

[18] Y. Ito, A. Saeki, K. Shiroyama, N. Sugioka and K. Takada, "Percutaneous Absorption of Interferon-A by SelfDissolving Micropiles," Journal of Drug Targeteting, Vol. 16, No. 3, 2008, pp. 243-249. doi:10.1080/10611860801902575

[19] Y. Ito, Y. Ohashi, A. Saeki, N. Sugioka and K. Takada, "Antihyperglycemic Effect of Insulin from Self-Dissolving Micropiles in Dogs," Chemical and Pharmaceutical Bulletin, Vol. 56, No. 3, 2008, pp. 243-246. doi:10.1248/cpb.56.243

[20] K. Takada, "Microfabrication Derived DDS: From Batch to Individual Production," Drug Discoveries and Therapeutics, Vol. 2, No. 3, 2008, pp. 140-155.

[21] Y. Ito, R. Hasegawa, K. Fukushima, N. Sugioka and K. Takada, "Self-Dissolving Microarray Chip as Percutaneous Delivery System of Protein Drug," Biological and Pharmaceutical Bulletin, Vol. 33, No. 4, 2010, pp. 683690. doi: $10.1248 / \mathrm{bpb} .33 .683$

[22] K. Fukushima, A. Ise, H. Morita, R. Hasegawa, Y. Ito, N. 
Sugioka and K. Takada, "Two-Layered Dissolving Microneedles for Percutaneous Delivery of Peptide/Protein Drugs in Rats," Pharmaceutical Research, Vol. 28, No. 1, 2011, pp. 7-21. doi:10.1007/s11095-010-0097-7

[23] G. Rozis, S. Silva, A. Benlahrech, T. Papagatsias, J. Harris, F. Gotch, G. Dickson and S. Patterson, "Langerhans Cells Are More Efficiently Tranduced Expressing Either Group C or B Fibre Protein: Implications for Mucosal Vaccines," European Journal of Immunology, Vol. 35, No. 9, 2005, pp. 2617-2626. doi:10.1002/eji.200425939

[24] V. Flacher, C. H. Tripp, P. Stoitzner, B. Haid, S. Ebner, B. D. Frari, F. Koch, C. G. Park, R. M. Steinman, J. Idoyaga and N. Romani, "Epidermal Langerhans Cells Rapidly Capture and Present Antigens from C-Type Lectin-Targeting Antibodies Deposited in the Dermis," Journal Investigative Dermatology, Vol. 130, 2010, pp. 755-762. doi:10.1038/jid.2009.343

[25] M. Pearton, S. Kang, J. Song, Y. Kim, F. Quan, A. Anstey, M. Ivory, M. R. Prausnitz, R. W. Compans and J. C. Birchall, "Influenza Virus-Like Particles Coated onto Micronedles Can Elicit Stimulatory Effects on Langerhans Cells in Human Skin," Vaccine, Vol. 28, No. 37, 2010, pp. 6104-6113. doi:10.1016/j.vaccine.2010.05.055

[26] K. Furmanov, M. Elnekave, D. Lehmann, B. E. Clausen, D. N. Kotton and H. Hovav, "The Role of Skin-Derived Dendric Cells in $\mathrm{CD}^{+} \mathrm{T}$ Cell Priming Following Immunization with Lentivectors," The Journal of Immunology, Vol. 184, No. 9, 2010, pp. 4889-4897. doi:10.4049/jimmunol.0903062

[27] N. Romani, M. Thurnher, J. Idoyaga, R. M. Steinman and V. Flacher, "Targeting of Antigens to Skin Dendritic Cells: Possibilities to Enhance Vaccine Efficacy," Immunology and Cell Biology, Vol. 88, 2010, pp. 424-430. doi:10.1038/icb.2010.39

[28] T. Jakob and M. C. Udey, "Epidermal Langerhans Cells: From Neurons to Nature's Adjuvants," Advanced Dermatology, Vol. 14, 1999, pp. 209-258.

[29] R. C. Yu, D. Abrams, M. Alaibac and A. C. Chu, "Morphological and Quantitative Analyses of Normal Epidermal Langerhans Cells Using Confocal Scanning Laser Microscopy," British Journal of Dermatology, Vol. 131, No. 6, 1994, pp. 843-848. doi:10.1111/j.1365-2133.1994.tb08587.x

[30] G. Murphy, D. Messadi, E. Fonferko and W. Hancock, "Phenotypic Transformation of Macrophages to Langerhans Cells in the Skin," American Journal of Pathology, Vol. 123, No. 3, 1986, pp. 401-406.
[31] A. N. Monteiro-Riviere, G. D. Bristol, O. T. Manning, A. R. Roger and E. J. Riviere, "Interspecies and Interregional Analysis of the Comparative Histologic Thickness and Laser Doppler Blood Flow Measurements at Five Custaneous Sites in Nine Species," Journal Investigative Dermatology, Vol. 95, 1990, pp. 582-586. doi:10.1111/1523-1747.ep12505567

[32] J. Bauer, A. F. Bahmer, J. Worl, W. Neuhuber, G. Schuler and M. Fartasch, "A Strikingly Constant Ration Exists between Langerhans Cells and Other Epidermal Cells in Human Skin. A Stereologic Study Using the Optical Dissector Method and the Confocal Laser Scanning Microscope," Journal Investigative Dermatology, Vol. 116, 2001, pp. 313-318. doi:10.1046/j.1523-1747.2001.01247.x

[33] L. M. J. Knippels, A. H. Penninks, M. Meeteren and G. F. Houben, "Humoral Cellular Immune Responses in Different Rat Strains on Oral Exposure to Ovalbumin," Food and Chemical Toxicology, Vol. 37, No. 8, 1999, pp. 881888. doi:10.1016/S0278-6915(99)00064-2

[34] T. K. Bhattacharyya, "Skin Aging in Animal Models: Histological Perspective,” In: M. A. Farage, K. W. Miller and H. I. Maibach, Eds., Textbook of Aging Skin, Springer Press, Berlin, 2010, pp. 5-12.

[35] L. B. S. Gerlinck, B. J. F Bemt, W. A. F Marijt, A. F. Biji, L. G. Visser, H. A. Cats, G. F. Rimmelzwaan and F. P. Kroon, "Intradermal Influenza Vaccination in Immunocompromized Patients Is Immunogenic and Feasible," Vaccine, Vol. 27, No. 18, 2009, pp. 2469-2474. doi:10.1016/j.vaccine.2009.02.053

[36] Y. Ito, T. Yamazaki, N. Sugioka and K. Takada, "SelfDissolving Micropile Array Tips for Percutaneous Administration of Insulin," Journal of Materials Science: Materials in Medicine, Vol. 21, No. 2, 2010, pp. 835-841. doi:10.1007/s10856-009-3923-X

[37] J. P. Caspers, W. G. Lucassen, A. H. Bruining and J. G. Puppels, "Automated Depth-Scanning Confocal Raman Microspectrometer for Rapid in Vivo Determination of Water Concentration Profiles in Human Skin," Journal of Raman Spectroscopy, Vol. 31, 2000, pp. 813-838.

[38] G. Widera, J. Johnson, L. Kim, L. Libiran, K. Nyam, P. E. Daddona and M. Cormier, "Effect of Delivery Parameters on Immunization to Ovalbumin Following Intracutaneous Administration by a Coated Microneedle Array Patch System," Vaccine, Vol. 24, No. 10, 2006, pp. 1653-1664. doi:10.1016/j.vaccine.2005.09.049 